\title{
Incidental finding of an arachnoid cyst in a patient presenting with features of postural headache after spinal anesthesia
}

\author{
Sang Hyun Lee ${ }^{1}$, Jin Gu Kang ${ }^{2}$, Woo Jong $\mathrm{Cho}^{2}$, Kyungmi Kim², and Jeong Heon Park ${ }^{2}$ \\ Department of Anesthesiology and Pain Medicine, ${ }^{1}$ Samsung Seoul Hospital, Sungkyunkwan University School of Medicine, Seoul, \\ ${ }^{2}$ Dongtan Sacred Heart Hospital, Hallym University College of Medicine, Hwasung, Korea
}

Postural headache can be caused by several underlying etiologies [1], therefore, when postural headache develops after spinal anesthesia, differential diagnosis and evaluation are imperative, despite a clear history of dural puncture and prominent features of headache with postural changes, indicating postdural puncture headache (PDPH). In this report, we address an incidental finding of an arachnoid cyst in a previously healthy, asymptomatic patient who experienced postural headache after spinal anesthesia for urologic surgery.

A 26 -year-old male $(180 \mathrm{~cm}, 68.8 \mathrm{~kg})$ was admitted for left varicocelectomy. He had not had any previous history of headache, neurologic signs or back pain, and his preoperative evaluation was normal. Thus spinal anesthesia was performed. For spinal anesthesia, a 25 gauge whitacre spinal needle was inserted at L3-4 via midline approach. The intrathecal space was approached at an initial attempt, and $0.5 \%$ bupivacaine (heavy Marcaine $^{\circledR}$, Astra Zeneca, Luton, UK) 14 mg with fentanyl 15 $\mu \mathrm{g}$ was injected slowly after confirming a free flow of the cerebrospinal fluid (CSF). There was no paresthesia or neurologic complication immediately after the procedure. After testing the spinal dermatome level up to the thoracic eight (T8), the surgery proceeded. After the surgery, the patient was transferred to the recovery room, and was sent to the ward after the dermatomal level dropped to thoracic level ten (T10). He did not complain of any discomfort or medical attention.

At the ward, the patient had lain supine for 8 postoperative hours to prevent PDPH. The patient admitted to having a headache upon sitting after 8-10 hours postoperatively. Hence, he was given oral analgesics, acetaminophen $650 \mathrm{mg}$ (Tylenol ${ }^{\circledR}$ ER TAB 650 mg, Janssen Korea Ltd, Seoul, Korea) three times per day. He was discharged on postoperative day 2 because he complained of only a mild headache.

Upon an outpatient urologic clinic visit two days after discharge, the patient complained of continued headache despite oral analgesics, acetaminophen $650 \mathrm{mg}$ (Tylenol ${ }^{\circledR}$ ER TAB $650 \mathrm{mg}$, Janssen Korea Ltd, Seoul, Korea) with postural aggravation. In a neurologist's opinion, he was suspected to have PDPH after spinal anesthesia; a brain computed tomography image was taken, which revealed an arachnoid cyst posterior to the cerebellum (Fig. 1). A neurosurgical opinion was consulted to assess the possible increase of intracranial pressure (ICP) owing to the cyst and determine whether any intervention was needed. The patient did not manifest any neurologic abnormality or a deficit of cranial nerve abnormality. The neurosurgical and neurologic consultation concluded that a low ICP was accounted for his headache, and that a conservative management may suffice. An epidural blood patch was also recommended if the headache persisted. The patient came back to the outpatient clinic 4 days after and he reported a complete recovery from his headache.

In the revised International Classification of Headache Disorders criteria, postural headache is defined as a headache occurring or worsening within 15 minutes after posing in the upright position and disappearing or resolving within 15 to 30 minutes after lying down [2]. Postural headache is usually caused by low ICP due to lumbar puncture, cerebrospinal fluid fistula, shunt overdrainage, or spontaneous intracranial hypotension [1].

Corresponding author: Jin Gu Kang, M.D., Ph.D., Department of Anesthesiology and Pain Medicine, Dongtan Sacred Heart Hospital, Hallym University College of Medicine, 7, Keunjaebong-gil, Hwaseong 445-170, Korea. Tel: 82-31-8086-2282, Fax: 82-31-8086-2029, E-mail: jg1229kr@hanmail.net (c) This is an open-access article distributed under the terms of the Creative Commons Attribution Non-Commercial License (http:// creativecommons.org/licenses/by-nc/3.0/), which permits unrestricted non-commercial use, distribution, and reproduction in any medium, provided the original work is properly cited. 

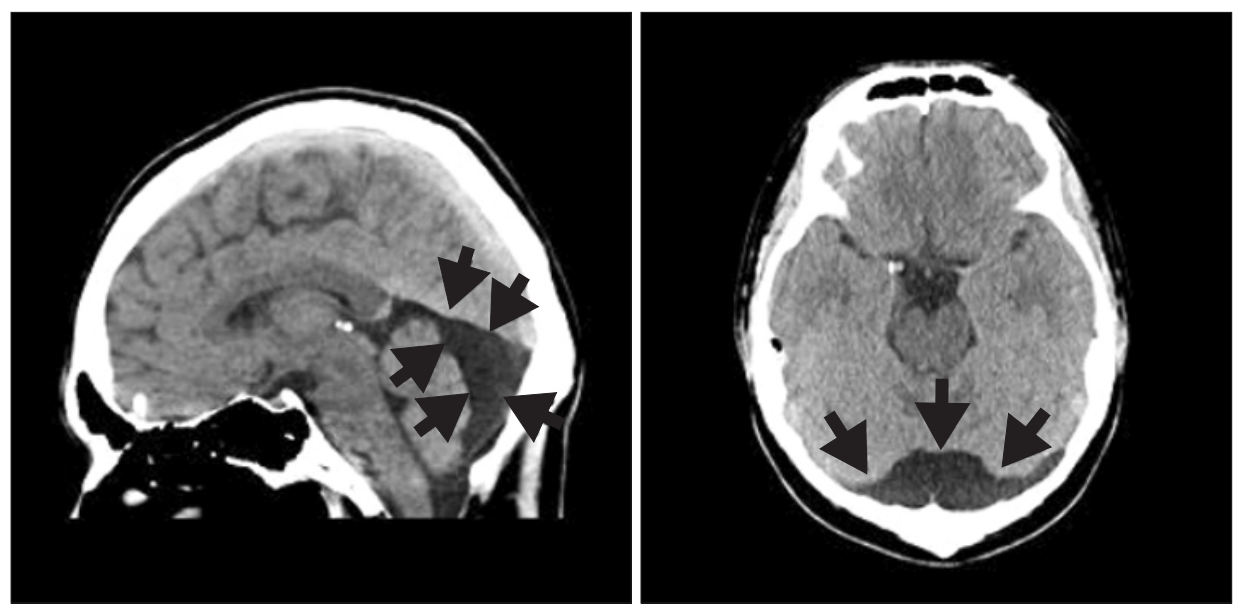

Fig. 1. A computed tomography (CT) scan showing an arachnoid cyst at posterior cranial fossa.

There are other reports of rare etiologies for postural headache that are unrelated to low ICP, such as herniation of a giant posterior fossa arachnoid cyst [1] or cerebellar hemorrhage [3]. In our patient, clinical features were that of $\mathrm{PDPH}$, however, upon imaging of the brain, an arachnoid cyst was incidentally found.

Arachnoid cysts are developed within or duplicated from the arachnoid membrane, and are filled with the CSF. They can suddenly rupture and intracystic hemorrhage may occur during changes in ICP, trauma or spontaneously. The size of the cysts may increase by a one-way valve system. Arachnoid cysts may also expand with a sudden decrease in CSF pressure near the cyst [4]. However, we were not sure if the low ICP headache was associated with the arachnoid cyst. There is no reported evidence whether arachnoid cysts can accentuate or precipitate PDPH after spinal anesthesia. It is not fully known whether spinal anesthesia can cause cyst expansion or precipitate the occurrence of symptoms due to changes in the CSF pressure profile. However, several reports present cases of intracranial or spinal arachnoid cyst after administration of safe spinal anesthesia in those patients who did not show signs of increased ICP [4].

In cases of postural headache after spinal anesthesia, the first impression is low ICP headache associated with PDPH and conservative treatment is practiced with neurological consultation. Most low ICP headaches are resolved spontaneously or respond to bed rest, intravenous hydration, and analgesics. An autologous blood patch can be considered if 24 hours of conservative treatment does not suffice. However, it should be kept in mind that postural headache may also be caused by other diseases such as cerebellar hemorrhage or herniation of giant posterior fossa arachnid cyst [1,3]. In this context, the value of neuroimaging and differential diagnosis in patients who develop postural headaches after spinal anesthesia should not be underestimated. A relatively high incidence of incidental arachnoid cysts in the general population and other possible causes of postural headache other than PDPH should motivate clinicians to verify otherwise, even in patients with clinically prominent postural headaches.

In considering intracranial pathology, it should be kept in mind that intracranial hypotension can also lead to intracranial hemorrhage through tearing of the bridging dural veins [5]. PDPH also needs to be differentiated from headache associated with pneumocephalus. PDPH can develop via two mechanisms; cerebrospinal fluid leakage (late onset, long lasting) and intrathecal air (immediate onset, short duration). In association with the latter mechanism, pneumocephalus may also develop from spinal anesthesia in rare cases, although most occur in epidural anesthesia.

In conclusion, clinicians including anesthesiologists must be aware that postural headaches need to be investigated with imaging workup and other potential underlying causes must be considered before making a final diagnosis of PDPH. While conservative treatments are practiced, early imaging workup must be considered for accurate diagnosis. 


\section{References}

1. Lu KC, Chao CC, Wang TL, Chong CF, Chen CC. A differential diagnosis in postural headache: herniation of a giant posterior fossa arachnoid cyst. Am J Emerg Med 2008; 26: 247.

2. Headache Classification Subcommittee of the International Headache Society. The International Classification of Headache Disorders: 2nd edition. Cephalalgia 2004; 24 Suppl 1: 9-160.

3. Chen WT, Fuh JL, Lu SR, Wang SJ. Cerebellar hemorrhage presenting as orthostatic headache: two case reports. Neurology 1999; 53: 1887-8.

4. Brice A, Barnichon C, Benhamou D. Intracranial arachnoid cysts and obstetric anesthesia: two case reports. Ann Fr Anesth Reanim 2010; 29: 648-50.

5. Chotai S, Kim JH, Kim JH, Kwon TH. Brain herniation induced by drainage of subdural hematoma in spontaneous intracranial hypotension. Asian J Neurosurg 2013; 8: 112-5. 